\title{
Hormonodependencia de la reacción de Arias Stella
}

\author{
Fabio Sánchez*; Antonio González**; Gonzalo Angel***; Mario Correa****; Constanza Díaz*****
}

\section{RESUMEN}

INTRODUCCION: La reacción de Arias Stella (RAS) se describió en el endometrio secretor, desde hace más de 4 décadas. Esta reacción se asoció inicialmente a embarazos intra y extrauterinos, pero posteriormente se describió RAS con el uso de citrato de clomifeno y progestágenos orales.

Se han efectuado sólo 2 estudios experimentales en animales inferiores sin que se haya encontrado una explicación única a este fenómeno.

Se programó una investigación en humanos, con el interés de clarificar su origen, y además explicar la presencia de este fenómeno, y asociado a la acción de la gonadotropina coriónica humana (HCG) o a la de un progestágeno, en este caso se utilizó la Gonadotropina Coriónica Humana y la 17 alfa hidroxiprogesterona, hormonas que actuarían sobre el endometrio proliferado, el cual fue obtenido artificialmente con la aplicación del valerianato de estradiol.

MATERIAL Y METODOS: Se seleccionaron 20 mujeres en la etapa de la menopausia, quienes presentaban niveles de FSH mayores de $18 \mathrm{ng} / \mathrm{ml}$ y estradiol menor de $30 \mathrm{pg} / \mathrm{ml}$, y quienes estaban programadas para histerectomía abdominal o vaginal por causa orgánica; las pacientes fueron sometidas a terapia estrogénica durante 35 días. Fueron divididas en $\mathbf{4}$ grupos para la aplicación hormonal así: grupo I, $500 \mathrm{mg}$ de 17 alfa hidroxiprogesterona cada 7 días 3 dosis grupo II, $1000 \mathrm{mg}$ de 17 alfa hidroxiprogesterona cada 7 días 3 dosis grupo III, $500 \mathrm{mg}$ de 17 alfa hidroxiprogesterona cada 7 días 3 dosis y gonadotropina coriónica humana 5000 UI cada 2 y $1 / 2$ días por 7 dosis; y grupo IV $500 \mathrm{mg}$ de 17 alfa hidroxiprogesterona cada 7 días 3 dosis y gonadotropina coriónica humana 1000 UI cada 2 y $1 / 2$ días por 7 dosis.

A todas las pacientes se les practicó histerectomía y a la pieza quirúrgica se le tomaron muestras de endometrio de áreas seleccionadas. Además se hicieron determinaciones hormonales para FSH, estradiol, 17 alfa hidroxiprogesterona y gonadotropina coriónica humana.

RESULTADOS: Se pudo apreciar la presencia de la RAS en 10 pacientes, independientemente de los 4 grupos seleccionados. Cuando se dividieron los pacientes entre los que tenían RAS presente y RAS ausente, se apreció como en el grupo de pacientes con RAS presente había mayor maduración del endometrio y se asoció a mayor concentración de los niveles séricos de 17 alfa hidroxiprogesterona, estadísticamente significativa, de $0,478 \pm 0.05$ en el grupo de RAS, contra $0,335 \pm 0.035$ en ausencia de RAS, con una $P$ de 0.0208 . No hubo diferencia significativa con las demás hormonas.

DISCUSION: Con los resultados de la presente investigación se puede concluir que la RAS es la respuesta a la acción de hormonas con actividad progestacional, hormonas que actúan sobre un endometrio proliferativo inducido por los estrógenos y con un elevado índice de maduración, fenómeno que es independiente de la existencia de embarazo.

PALABRAS CLAVES: Endometrio, Arias Stella, Humanos.

\section{SUMMARY}

INTRODUCTION: ARIAS-STELLA REACTION (ASR) was described on the secretor endometrium since more than 40 years ago and it was associated initially to intra and extra-uterine pregnancies, but later was also observed with the use of Clomiphen-Citrate and oral progestogens.

Only 2 experimental studies have been carried out in lower animals without finding a clear explanation for that phenomenon.

A research in humans was programmed in order to explain and clarify the ASR origin and that to action of the human chorionic gonadothropine (HCG) or a progestogen. In this case HCG and 17 alpha hydroxi progesterone were used to act on the proliferated endometrium which previously was artificialy obtained by using estradiol valerianate.

MATERIAL AND METHODS: 20 menopausic women with FSH levels above $18 \mathrm{ng} / \mathrm{ml}$, estradiol levels below $30 \mathrm{pg} / \mathrm{ml}$ and programmed for vaginal or abdominal histerectomy for organic cause were selected. This patient were submitted to estrogenic therapy for 35 days. They were distributed the following $\mathbf{4}$ groups for the hormonal application.

\footnotetext{
* Profesor Titular Depto. de Obstetricia y Ginecología.

** Residente Depto. de Obstetricia y Ginecologia.

*** Residente Depto. de Patología.
}

**** Profesora Titular Depto. de Patología.

***** Departamento de Obstetricia y Ginecología y Patología. Facultad de Medicina, Universidad de Antioquia Medellín, Colombia. 
Group I: $500 \mathrm{mg}$ - 17 alpha $\mathrm{OH}$ progesterone caproate every 7 days, 3 doses. Group II: $1.000 \mathrm{mg}$ - 17 alpha OH progesterone caproate every 7 days, 3 doses. Group III: $500 \mathrm{mg}$ - 17 OH progesterone caproate every 7 days, 3 doses, and HCG 5.000 UI every $21 / 2$ days for 7 doses. And Group IV: $500 \mathrm{mg}$ - 17 alpha OH progesterone every 7 days, 3 doses, and HCG 10.000 UI every $2 \frac{1}{2}$ days for 7 doses.

Al the patients were histerectomized and endometrium samples from selected areas of the surgical especimens were taken. In addition hormonal determinations for FSH, estradiol, 17 alpha $\mathrm{OH}$ progesterone and HCG were made.

RESULTS: The was observed in ten patients without regards from which of the four groups they came. When the patients were divided between ASR + and ASR-groups, it was observed that the ASR + patients group, there was greater endometrium maturation and it was associated to higher seric levels of 17 alpha $\mathrm{OH}$ progesterone, statistically significant $0,478 \pm 0.05$ in the ASR + group versus $0.335 \pm 0.035$ in the ASR -group, $p=0.0208$.

There was no significant differences between the other hormones.

DISCUSSION: The above results suggest that ASR is the response to the action of progestational hormones wich act on the estrogen induced proliferating endometrium with a raised maturation index, independent to and existing pregnancy.

KEY WORDS: Endometrium, Arias Stella, humanos

\section{Introducción}

Hace cerca de 4 décadas fue descrito el fenómeno de Arias Stella (RAS) (1), y desde entonces existe controversia respecto a su importancia clínica y a su etiología. En la literatura no se encontraron estudios dirigidos a medir su sensibilidad y especificidad diagnóstica con respecto al embarazo. Además las investigaciones experimentales dirigidas a explicar este fenómeno sólo se han efectuado en animales inferiores (2-3).

La existencia de la RAS fue descrita inicialmente en pacientes con aborto incompleto, enfermedad trofoblástica gestacional, endometritis sincitial y embarazo ectópico (1-4), pero con el tiempo también se describió en pacientes con embarazo normal (5), o como respuesta a la ingesta de citrato de clomifeno (4), progestágenos orales (6), y aún en el ciclo normal.

Son múltiples las teorías con respecto a su patogenia, las investigaciones experimentales en animales inferiores son contradictorias y existe controversia acerca del fenómeno, si es un proceso dinámico del endometrio (5-7) o por el contrario es una manifestación regresiva (8).

Interesados en clarificar estos interrogantes que aunque no son de actualidad si son motivo de controversias periódicamente, y con el ánimo de definir el papel de la RAS en el proceso de la reproducción humana se planeó esta investigación experimental en humanos.

\section{Material y métodos}

Pacientes: Se seleccionaron 20 pacientes que consultaron al servicio de Obstetricia y Ginecología, Hospital Universitario San Vicente de Paúl, Medellín, durante el año de 1990, y quienes fueron programadas para histerectomía por enfermedad orgánica como prolapso genital, o neoplasia intraepitelial, y que estuvieran por más de 1 año en menopausia, con niveles plasmáticos de FSH mayores de $18 \mathrm{ng} / \mathrm{ml}$ y Estradiol menores de $30 \mathrm{pg} /$ $\mathrm{ml}$. Ninguna paciente ingirió terapia hormonal de sustitución en los últimos tres meses.
Se seleccionaron pacientes menopaúsicas con el fin de eliminar la acción de hormonas endógenas (estrógenos, andrógenos o progesterona) producidas en el ovario que pudieran actuar como factor perturbador y poder lograr así obtener modificaciones en el endometrio, secundarias a la terapia hormonal exógena (9).

A cada una de las pacientes se le informó individualmente acerca de los objetivos, programación, efectos secundarios y riesgos de la investigación; además se les dejó en libertad de aceptar o retirarse del programa. Cada una de las pacientes firmó un documento que autorizaba la investigación.

\section{Metodología}

Las pacientes fueron divididas en 4 grupos de acuerdo al esquema de aplicación de las drogas y fueron incluidas en cada grupo de acuerdo al orden de llegada. Inicialmente se programó el grupo I, luego el II, III, y al final el IV, con el fin de facilitar el control de los equemas.

Esquema terapéutico: Antes de iniciar la investigación se informó a la paciente los medicamentos que debía ingerir, y las fechas de toma de muestras en el servicio. A todas las pacientes se le inició tratamiento con $2 \mathrm{mg}$ de valerianato de estradiol (Progynova ${ }^{R}$ ), siguiendo el mismo esquema que se utiliza para pacientes con disgenesia gonadal en los programas de fertilización in vitro, con el fin de obtener un endometrio proliferativo avanzado (10), así:

. 1 comprimido $(2 \mathrm{mg}) / 1$ al 10 días.

.3 comprimidos $(6 \mathrm{mg}) / 11$ al 14 días.

.1 comprimido $(2 \mathrm{mg}) / 15$ al 18 días.

. 2 comprimidos $(4 \mathrm{mg}) / 19$ al 35 días.

El día 15 del ciclo se le aplicó a cada paciente $250 \mathrm{mg}$ de caproato de 17 alfa hidroxi-progesterona IM (Proluton ${ }^{\mathrm{R}}$ ), con el fin de desencadenar una respuesta secretora en el endometrio, compatible con la del día 21 del ciclo. Posteriormente, dentro de 7 días se administraron esquemas terapéuticos diferentes de acuerdo al grupo a que pertenecen así: 
Grupo I $500 \mathrm{mg}$ de caproato de 17 alfa hidroxiprogesterona (17 OPH) IM cada 7 días, 3 dosis.

Grupo II $1000 \mathrm{mg}$ de caproato de $17 \mathrm{OPH}$ cada 7 días, 3 dosis.

Grupo III $500 \mathrm{mg}$ de caproato de $17 \mathrm{OPH}$ cada 7 días, 3 dosis y 5.000 UI de Gonadotropina Coriónica Humana (Promogonil ${ }^{\mathrm{R}}$, HCG) IM cada 2 y $1 / 2$ días por 7 dosis.

Grupo IV $500 \mathrm{mg}$ de caproato de $17 \mathrm{OPH}$ cada 7 días, 3 dosis y 10000 UI de HCG cada 2 y $1 / 2$ días por 7 dosis.
La dosis de valerianato de estradiol se le entregó a la paciente con sus respectivas instrucciones. En la segunda fase del ciclo se les aplicó en la institución, la ampolla de 17 hidroxiprogesterona, los días 15,22 y 29 y la HCG los días $19,21,24,26,29,31$ y 34 del ciclo artificial. Todas las aplicaciones fueron intramusculares a las $9 \mathrm{am}$., excepto la HCG los días 21, 26 y 31, la cual se aplicó a las 21 horas en la residencia de la paciente y quien con posterioridad debía traer la respectiva ampolla vacía como control de su aplicación (Figura 1). Todo el esquema terapéutico fue supervisado permanentemente por uno de los autores.

Figura 1

\section{ESQUEMA DE APLICACION DE LOS DIFERENTES MEDICAMENTOS}

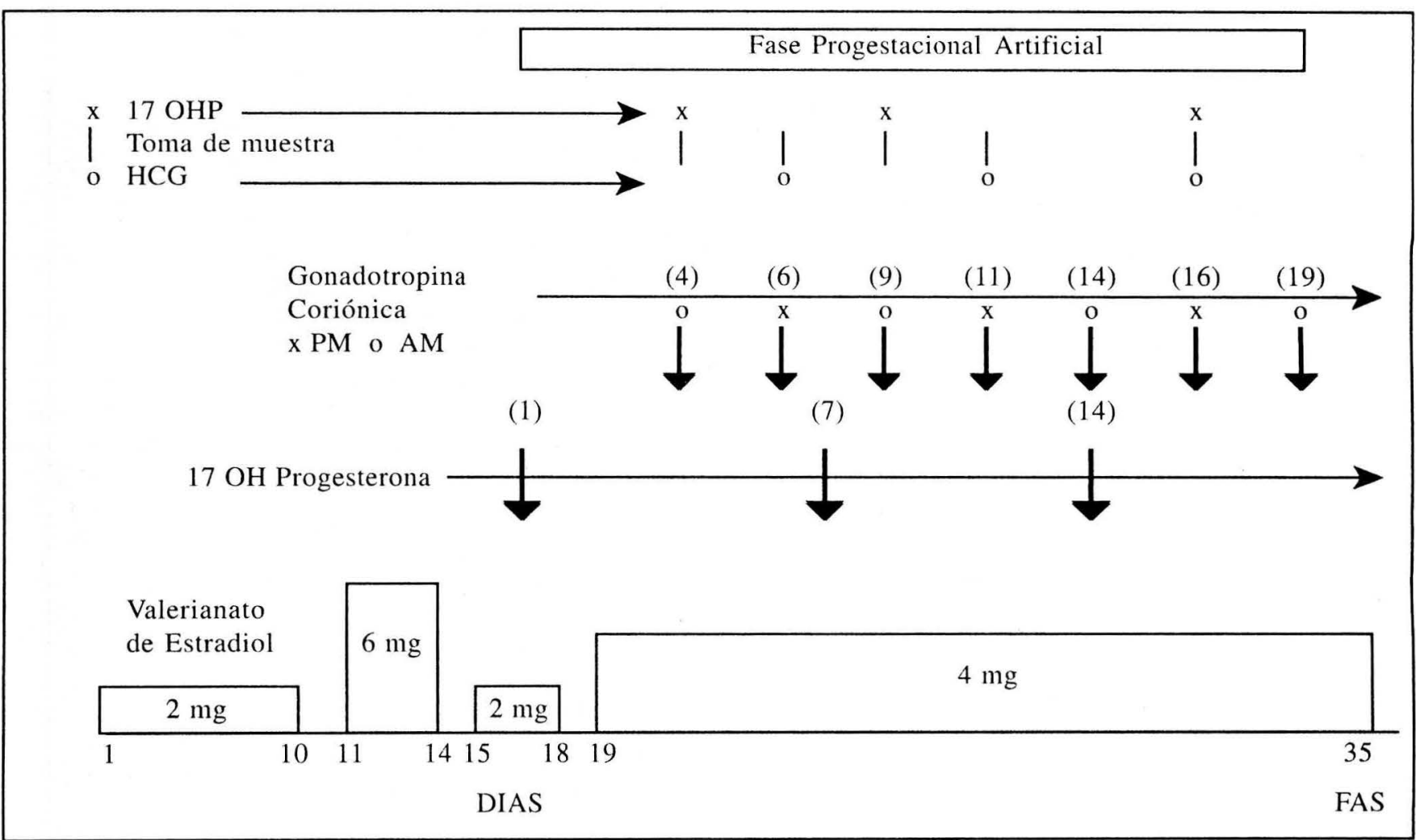

Control hormonal: La respuesta hormonal fue monitorizada por medición de niveles séricos, para ello se tomaron muestras de $10 \mathrm{cc}$ de sangre de la vena cubital anterior, los días $19,24,26,29$ y 33, la sangre se almacenó en tubo seco, se dejó 1 hora en reposo hasta obtener la retracción del coágulo; el plasma se fraccionó en 2 alícuotas y se conservó en refrigeración a $-20^{\circ} \mathrm{C}$ hasta su procesamiento. Todos los sueros se procesaron al término de la investigación.

Control histológico: El día 35 del ciclo artificial, se les practicó a cada una de las pacientes histerectomía y salpingectomía total o parcial; el especimen quirúrgico se conservó en alcohol isopropílico al $90 \%$ o en formol neutro hasta su procesamiento.

El útero se seccionó lateralmente hasta el fondo, previa amputación del cuello. Se tomaron bloques longitudinales de 2 a $3 \mathrm{~mm}$ cada centímetro tanto en la cara anterior como en la posterior, lo mismo ocurrió en el canal cervical. Estos cortes comprenden todo el espesor desde la mucosa hasta la serosa y de zonas anormales específicas a la evaluación macroscópica. De la trompa se tomaron 3 muestras (proximal, medial y distal) y cortes específicos de zonas anormales. Los tejidos fueron fijados y coloreados con hematoxilina eosina.

La lectura del endometrio se efectuó en forma independiente por 2 patólogos, siguiendo parámetros acordados previamente y después de definir como criterios diagnósticos de la RAS: aumento del número de glándulas con actividad secretora, disminución del calibre de la luz con contenido de secreción, incremento de su vascularización estromal, células epiteliales con vacuolización citoplasmática, incremento del citoplasma con 
relación al núcleo, pérdida del borde celular, núcleos hipercromáticos e hipertróficos, con tendencia a la multilobulación y mitosis atípicas en el estroma, reacción decidual.

A cada uno de los elementos endometriales se le dio un puntaje con el fin de definir un índice de maduración, para lo cual se dividió el tejido en glándulas y estroma.

La maduración glandular se clasificó en:

a) Respuesta luminal: Luz no colapsada: 1, Colapsada: 2

b) Respuesta epitelial: Vacuolas ausentes: 1, Subnucleares: 2, Supranucleares: 3, Exhausta: 4.

c) Respuesta nuclear: Ovales: 1, redondos: 2, poligonales: 3, bizarros: 4 .

d) Atipia nuclear: ausentes: 1, escasas: 2, frecuentes: 3, abundantes: 4 .

Se obtiene así un puntaje máximo de 14 .

La maduración estromal se clasificó siguiendo los siguientes parámetros:

a) Predecidualización: predecidua ausente: 1, perivascular: 2 , periglandular: 3 y total: 4 .

b) Respuesta granulocítica: Granulocitos ausentes: 1, perivascular: 2, difusos: 3 , abundantes: 4 .

c) Hemorragias: ausente 1, perivascular: 2, confluentes: 3 , total: 4 .

d) Edema: ausente: 1, leve: 2, moderado: 3, severo: 4 .

e) Respuesta vascular: Vasos ausentes: 1, presentes: 2, hiperplásicos en capa media: 3.

Se obtiene así un puntaje máximo de maduración estromal de 19 puntos y el total de maduración endometrial de 33 puntos. Con los puntajes anteriores se obtienen 3 índices de maduración: glandular que resulta de dividir el puntaje total glandular del endometrio del paciente por 14 , el estromal se divide por 19 y el endometrial por 33 .

Tanto los patólogos como los analistas desconocían el esquema terapéutico al cual se había sometido la paciente, este sólo se conoció al momento de tener el informe estadístico, conformándose así un estudio doble ciego.

Determinaciones hormonales: Las hormonas FSH, $\mathrm{HCG}$, Estradiol $\left(\mathrm{E}_{2}\right)$ y $17 \mathrm{OPH}$ fueron determinadas por RIA, suministrado por Diagnostic Products Corp (Los Angeles CA), todas las determinaciones se hicieron con el mismo estuche y en el mismo laboratorio.

La mínima dosis detectable para FSH es $0.1 \mathrm{mUI} / \mathrm{ml}$, de HCG $1,1 \mathrm{mUI} / \mathrm{ml}$, estradiol $8 \mathrm{pg} / \mathrm{ml}$ y 17 hidroxiprogesterona $0.07 \mathrm{ng} / \mathrm{ml}$, el coeficiente de variación (CV) fue de 1,63 para FSH, 5,13 para $\mathrm{HCG}, 4,35$ para $\mathrm{E}_{2}$ y 1,93 para la $17 \mathrm{OPH}$.

Análisis estadístico: Toda la información fue recopilada en un formulario previamente precodificado y los datos almacenados en computador NEC. Los análisis se hicieron por medio de un paquete estadístico, se utilizaron métodos descriptivos y en el estudio analítico, el método de ANOVA en dos vías.

\section{Resultados}

El promedio de edad de las 20 mujeres participantes en el estudio fue de 56 años, con un rango entre los 50 y
64 años. La indicación para la histerectomía fue por prolapso genital en 17 mujeres, y en 3 neoplasia intraepitelial cervical.

El promedio de FSH en las 20 pacientes fue 19,44 \pm $1.04 \mathrm{ng} / \mathrm{ml}$ y de $\mathrm{E}, 8.50 \pm 2.76 \mathrm{pg} / \mathrm{ml}$, niveles séricos que confirman el estado menopáusico de las pacientes, de acuerdo a los criterios establecidos.

Los efectos secundarios a la administración hormonal fueron mínimos (náuseas, epigastralgias, tensión mamaria), en ningún caso fue necesario la suspensión del mismo. Sólo se presentó una complicación postoperatoria (obstrucción intestinal por bridas) sin relación con las drogas administradas.

En todas las mujeres se observó con el tratamiento un incremento progresivo de los niveles séricos de $\mathrm{E}_{2}$, desde un promedio de $466,51 \pm 28.60 \mathrm{pg} / \mathrm{ml}$ el día 19 del ciclo hormonal hasta $638,87 \pm 101,27 \mathrm{pg} / \mathrm{ml}$ para el día 34 (tabla 1). El análisis de varianza con relación a los niveles de $E_{2}$ no mostró diferencia estadísticamente significativa $(\mathrm{p}<0.7418)$ en los 4 grupos (tabla 2$)$.

Al mismo tiempo, en todas las mujeres se observó un incremento progresivo en los niveles séricos de 17 alfa hidroxiprogesterona desde un promedio de $0.250 \pm 0.03$ $\mathrm{ng} / \mathrm{ml}$ para el día 19 del ciclo hormonal, hasta $0.561 \pm$ $0.071 \mathrm{ng} / \mathrm{ml}$ para el día 34 (tabla 1). El análisis de varianza con relación a los niveles séricos de 17 alfa hidroxiprogesterona mostró diferencia estadísticamente significativa $\mathrm{p}<0.00001$ entre los diferentes tiempos y de 0.0058 entre los pacientes del grupo II con respecto a los demás grupos experimentales (tabla 2).

Contrario a lo esperado, no se logró un incremento progresivo en los niveles séricos de HCG en los grupos III y IV y sólo se observó un descenso aunque no significativo a través del tiempo $(\mathrm{p}<0.5171)$ en los dos grupos (tabla 1).

La aplicación de una dosis mayor de HCG al grupo IV no logró incrementar el nivel sérico de esta hormona con respecto al grupo III, así $109,78 \pm 6.51 \mathrm{mUI} / \mathrm{ml}$ en el . grupo III vs $103,19 \pm 3.6 \mathrm{mUI} / \mathrm{ml}$ en el IV $(\mathrm{p}<0.4105)$ (tabla 2).

La RAS se observó en todos los grupos experimentales: 2 en el grupo I, 4 en el II, 3 en el III y 1 en el IV (Tabla $2)$. En general se obtuvo RAS en 10 pacientes (50\%), en 9 de ellas localizada en el fondo uterino y una con distribución difusa. Estas pacientes se agruparon entre sí con el fin de compararlas con el grupo sin RAS.

Al agrupar los niveles plasmáticos de las diferentes hormonas en las pacientes con ausencia o presencia de la R.A.S, se observó que no hubo diferencia estadísticamente significativa en los 2 grupos con respecto a los niveles de $E_{2}: 559,88 \pm 69,3 \mathrm{ng} / \mathrm{ml}$ vs $541.87 \pm 38,8 \mathrm{pg} / \mathrm{ml}(\mathrm{p}<$ $0.8245)$ ni con respecto a los niveles séricos de la HCG ( $p$ $<0.3666$ ) siendo las concentraciones de 103,66 \pm 4.6 $\mathrm{mUI} / \mathrm{ml}$ en el grupo con RAS y $110,71 \pm 6.23 \mathrm{mUI} / \mathrm{ml}$ en el grupo con ausencia de RAS. En tanto se observó diferencia significativa en los niveles plasmáticos de la 17 alfa hidrOxiprogesterona; las pacientes con RAS mostraron mayores niveles: $0.478 \pm 0.05 \mathrm{ng} / \mathrm{ml}$ contra $0.335 \pm 0.035 \mathrm{ng} / \mathrm{ml}$ en las pacientes con ausencia de RAS $(\mathrm{p}<0.0208)$ (tabla 3$)$. 
Tabla 1

CONCENTRACION DE LAS HORMONAS EN DIFERENTES TIEMPOS

\begin{tabular}{|c|c|c|c|c|c|c|}
\hline \multirow[b]{2}{*}{ Hormonas } & & \multicolumn{4}{|c|}{ Días } & \multirow[b]{2}{*}{ Valor de $\mathrm{P}$} \\
\hline & & 1 & +4 & +11 & +19 & \\
\hline $\begin{array}{l}\mathrm{E}_{2} \\
(\mathrm{Pg} / \mathrm{ml})\end{array}$ & $\begin{array}{l}x \\
\pm\end{array}$ & $\begin{array}{l}8.50 \\
2.75\end{array}$ & $\begin{array}{r}466.51 \\
28.60\end{array}$ & $\begin{array}{r}547.24 \\
50.76\end{array}$ & $\begin{array}{l}638.87 \\
101.27\end{array}$ & $0.00001 *$ \\
\hline $\begin{array}{l}17 \text { OHP } \\
(\mathrm{ng} / \mathrm{ml})\end{array}$ & $\begin{array}{l}x \\
\pm\end{array}$ & $\begin{array}{l}0.15{ }^{(1)} \\
0.20\end{array}$ & $\begin{array}{l}0.24 \\
0.03\end{array}$ & $\begin{array}{l}0.41 \\
0.04\end{array}$ & $\begin{array}{l}0.56 \\
0.07\end{array}$ & $0.0001 *$ \\
\hline $\begin{array}{l}\text { HCG } \\
(\mathrm{mUI} / \mathrm{ml}) \\
9-14-19 \text { Días }\end{array}$ & $\begin{array}{l}\mathrm{x} \\
\pm\end{array}$ & 0.00 & $\begin{array}{r}112.50 \\
7.25\end{array}$ & $\begin{array}{r}105.24 \\
7.12\end{array}$ & $\begin{array}{r}101.71 \\
4.77\end{array}$ & 0.5171 \\
\hline
\end{tabular}

* Diferencia Estadísticamente Significativa

(1) Tomado de Obstet. Gynecol. 1976; 48: 150.

Tabla 2

CONCENTRACION DE LAS DIFERENTES HORMONAS EN LOS DIFERENTES GRUPOS

\begin{tabular}{|c|c|c|c|c|c|}
\hline & \multicolumn{4}{|c|}{ Grupos } & \multirow[b]{2}{*}{ Valor de P. } \\
\hline $\begin{array}{l}\text { Determinación } \\
\text { hormonal }\end{array}$ & $\begin{array}{c}\mathrm{I} \\
\mathrm{n}=5\end{array}$ & $\underset{\mathrm{n}=5}{\mathrm{II}}$ & $\begin{array}{c}\text { III } \\
n=5\end{array}$ & $\begin{array}{c}\text { IV } \\
n=5\end{array}$ & \\
\hline $\mathrm{E}_{2}(\mathrm{Pg} / \mathrm{ml})$ & $\begin{array}{lr}x & 381.81 \\
\pm & 60.55\end{array}$ & $\begin{array}{r}472.63 \\
99.49\end{array}$ & $\begin{array}{r}396.18 \\
73.98\end{array}$ & $\begin{array}{r}410.51 \\
82.22\end{array}$ & 0.7418 \\
\hline $\begin{array}{l}17 \mathrm{OHP} \\
(\mathrm{ng} / \mathrm{ml})\end{array}$ & $\begin{array}{ll}x & 0.36 \\
\pm & 0.04\end{array}$ & $\begin{array}{l}0.56 \\
0.06\end{array}$ & $\begin{array}{l}0.41 \\
0.09\end{array}$ & $\begin{array}{l}0.28 \\
0.04\end{array}$ & $0.0058 *$ \\
\hline $\begin{array}{l}\mathrm{HCG} \\
(\mathrm{mUI} / \mathrm{ml})\end{array}$ & $\begin{array}{ll}x & ---- \\
\pm & \end{array}$ & ---- & $\begin{array}{r}109.78 \\
6.51\end{array}$ & $\begin{array}{r}103.19 \\
3.61\end{array}$ & 0.4105 \\
\hline $\begin{array}{l}\text { Reacción } \\
\text { A. Stella }\end{array}$ & 2 & 4 & 3 & 1 & \\
\hline
\end{tabular}

* Diferencia Estadísticamente Significativa

Tabla 3

CONCENTRACION DE LAS DIFERENTES HORMONAS DE ACUERDO A LA PRESENCIA

O AUSENCIA DE LA REACCION DE ARIAS STELLA

\begin{tabular}{|l|c|c|c|}
\cline { 2 - 4 } \multicolumn{1}{c|}{} & \multicolumn{2}{c|}{ Reacción de Arias Stella } & \multicolumn{1}{c}{ Valor de P. } \\
\hline $\begin{array}{l}\text { Determinación } \\
\text { Hormonal }\end{array}$ & $\begin{array}{c}\text { Presente } \\
\mathrm{n}=10\end{array}$ & $\begin{array}{c}\text { Ausente } \\
\mathrm{n}=10\end{array}$ & 0.8245 \\
\hline Estradiol $(\mathrm{pg} / \mathrm{ml})$ & $559.88 \pm 69.3$ & $541.87 \pm 38.8$ & 0.3666 \\
\hline $\begin{array}{l}\text { Gonadotropina } \\
\text { Coriónica }(\mathrm{mUI} / \mathrm{ml})\end{array}$ & $103.66 \pm 4.6$ & $110.71 \pm 6.23$ & $0.0208 *$ \\
\hline $\begin{array}{l}17 \text { Hidroxi } \\
\text { Progesterona }(\mathrm{ng} / \mathrm{ml})\end{array}$ & $0.478 \pm 0.05$ & $0.335 \pm 0.03$ & \\
\hline
\end{tabular}

* Diferencia Estadísticamente Significativa. 
El tipo de endometrio obtenido en todas las pacientes, fue secretor y decidualizado en 18. En las pacientes con RAS sólo una no presentaba reaccióm decidual.

Si se compara la distribución de frecuencias de los índices de maduración estromal y total de las pacientes con RAS con relación a las que no presentaban el fenómeno, no se aprecia diferencia significativa $(\mathrm{p}<0.1130$ y $\mathrm{p}$ $<0.3272$ respectivamente), en tanto se aprecia una diferencia significativa (Test de Fischer $p<0.0054$ ) en el índice de maduración glandular (tabla 4).

\section{Tabla 4}

\section{INDICE DE MADURACION ENDOMETRIAL}

\begin{tabular}{|l|c|c|c|c|c|c|}
\hline \multirow{2}{*}{$\begin{array}{l}\text { Indice de } \\
\text { Maduración }\end{array}$} & \multicolumn{2}{|c|}{ Glandular } & \multicolumn{2}{c|}{ Estromal } & \multicolumn{2}{c|}{ Total } \\
\cline { 2 - 7 } $\begin{array}{l}0.26-0.50 \\
0.51-0.75\end{array}$ & + & - & + & - & + & 1 \\
8 \\
4
\end{tabular}

* Diferencia Estadísticamente Significativa.

\section{Discusión}

Desde que se describió la RAS, se han publicado numerosos artículos clínicos que correlacionan su aparición con embarazo y con sus estados patológicos, pero a medida que avanzó la investigación clínica se describió su asociación con otros estados diferentes tal como el aborto incompleto, la enfermedad trofoblástica gestacional, la endometritis sincitial y el embarazo ectópico (1-4), perdiendo su validez como medio de diagnóstico. Sin embargo, a pesar de estas controversias, no se han efectuado investigaciones tendientes a aclarar su papel o a medir su sensibilidad y especificidad.

Las investigaciones experimentales que se han efectuado se hicieron en animales inferiores y son aparentemente contradictorias, pues mientras Arias Stella encontró la RAS con la aplicación de $\mathrm{E}_{2}$ y $\mathrm{HCG}$ en ratas con presencia de ovarios, y con la aplicación de $E_{2}$ y progesterona $(\mathrm{P})$ en ratas ooforectomizadas (2), Dallembach la encontró asociada a $\mathrm{E}_{2}$ y relaxina $(\mathrm{Rx})(3)$.

De acuerdo a las diferentes investigaciones se le ha atribuido la RAS al $\mathrm{E}_{2}, \mathrm{P}, \mathrm{HCG}$ y Rx, sin que se atribuya este fenómeno a una hormona específica, por lo cual seleccionamos estas hormonas para la investigación, excepto la relaxina, ya que se consideró que su secreción era secundaria a los cambios estromales desencadenados por la progesterona o los progestágenos, a nivel de los granulocitos estromales (11-12).

En cambio se aplicó la HCG y la $17 \mathrm{OPH}$, en diferentes dosis con el fin de tratar de asociar la presencia de la RAS con la concentración de una de estas hormonas. Se utilizó la $17 \mathrm{OPH}$ por ser una sustancia con propiedades muy semejantes a la progesterona y con una vida media mayor.

Todas estas aparentes inconsistencias en animales fueron el motivo para programar esta investigación en humanos, con el fin de lograr reproducir el fenómeno y tratar de lograr una explicación de su origen. Se seleccionaron mujeres menopáusicas con el fin de eliminar factores endógenos ( $E_{2}$ - P y Andrógenos) del ovario que modificaran la respuesta y poder así atribuir su presencia a una o varias hormonas exógenas. Todos los pacientes presentaron niveles de $\mathrm{E}_{2}$ y FSH compatibles con menopausia.

A todas las pacientes se les administró el mismo esquema de $\mathrm{E}_{2}$, utilizado en los programas de fertilización in vitro (9), con el fin de obtener un endometrio proliferativo capaz de responder a la acción secretora de la $17 \mathrm{OPH}$ y posiblemente de la HCG; fue así como con este esquema, todos los endometrios obtenidos fueron secretores, con algún grado de mayor o menor maduración glandular o estromal y los niveles séricos de $\mathrm{E}_{2}$ y $17 \mathrm{OPH}$ fueron muy semejantes a los encontrados durante el embarazo inicial.

No se obtuvieron niveles séricos pre tratamiento de $17 \mathrm{OPH}$ en las pacientes estudiadas, por lo cual se tomó como referencia los valores obtenidos por otros autores, lo que permite establecer que el promedio plasmático de la $17 \mathrm{OPH}$ en pacientes postmenopáusicas es de $0.15 \pm$ $0.02 \mathrm{ng} / \mathrm{ml}$ (9). Hormona que tiene un ritmo circadiano cuyos niveles plasmáticos más elevados ocurren alrededor de las 8 horas.

Pruebas de estimulación con ACTH y de supresión con dexametasona demuestran que en la menopausia la mayor producción de hormonas, es de origen adrenal, a pesar de que también viene en declinación (Adrenopausia). Los niveles plasmáticos de progesterona en este grupo de pacientes, es de $0.28 \pm 0.03 \mathrm{ng} / \mathrm{ml}$ la cual también es de origen suprarenal (12).

En esta investigación la aplicación parenteral de 17 $\mathrm{OPH}$ produjo un incremento significativo en los niveles 
séricos de esta hormona, alrededor de un $270 \%$ con respecto a la basal y similar a los niveles séricos encontrados durante la fase lútea.

A pesar de los diferentes esquemas terapéuticos, la RAS se encontró en todos los grupos, independiente de la presencia o ausencia de HCG y por el contrario su mayor frecuencia se apreció en el grupo II al cual se le había aplicado la mayor dosis de 17 OHP y cuyos niveles promedios en suero de $\mathrm{E}_{2}$ y $17 \mathrm{OHP}$ eran mayores que en los demás grupos.

A diferencia de lo que se esperaba encontrar, los grupos 3 y 4 a quienes se les administró HCG no presentaron RAS con mayor frecuencia y los niveles hormonales de $\mathrm{HCG}$, entre estos dos grupos no tenían diferencia significativa (Tabla 2). Los niveles séricos de HCG no tuvieron un incremento, posiblemente como consecuencia de su metabolismo, es así como la curva de HCG en suero después de una dosis parenteral determinada desencadena un pico aproximadamente a las 5,6 horas de aplicada y desaparece rápidamente en las siguientes 23 horas (13). Su titulación 2 y 1/2 días después presenta niveles entre 100 y $200 \mathrm{mUI} / \mathrm{ml}$, como se confirmó en la presente investigación, logrando reproducir los niveles séricos de HCG compatibles con un embarazo temprano (días 29 a 32 después de la última menstruación).

Al no encontrarse una evidente hormonodependencia de la RAS con respecto a los niveles de $\mathrm{HCG}$, se reorganizaron las pacientes en 2 grupos así: con presencia y ausencia de RAS, se estableció un análisis de su comportamiento hormonal. Se encontró que no existía ninguna diferencia significativa entre los 2 grupos con relación a los niveles de $\mathrm{E}$, y $\mathrm{HCG}$ en tanto, con relación a los niveles de $17 \mathrm{OPH}$ se apreció una diferencia significativa $(\mathrm{P}<0.0208)$ (Tabla 3), hallazgo que estaría en favor de explicar la presencia de la RAS secundariamente a la acción de un progestágeno (progesterona en el caso de las investigaciones de Arias Stella y 17 hidroxiprogesterona como en la presente investigación).

A raíz de estos hallazgos quedaría por explicar la presencia de la RAS en los estudios experimentales de Arias Stella en el grupo 1 cuando utiliza E, y HCG en ratas no castradas (2). Se postula que pudo haber ocurrido la ovulación en estos animales como consecuencia del tiempo de estudio de 28 días o por la utilización de HCG como así lo interpreta el autor, la presencia de cuerpo lúteo y por lo tanto de progesterona explicarían la RAS, siendo en este caso el papel de la HCG el de una hormona que estimula la ovulación y la luteogénesis en el ovario, y la progesterona, hormona que tendría el papel generador de la RAS al actuar sobre un endometrio proliferativo.

Un segundo experimento en ratas efectuado por Dallembach, aunque contradice los hallazgos anteriores y asigna a los estrógenos y/o a la relaxina un papel en la R.A.S., observó cambios compatibles con RAS en el endometrio de ratas normales sometidas a $\mathrm{HCG}$ sola o en combinación con relaxina y en ratas castradas sometidas a estrógenos solos o en combinación con $\mathrm{HCG}$ o relaxina (3).

Dallenbach concluye que: los cambios endometriales son provocados por la hiperactividad de la $\mathrm{HCG}$ que induce al ovario a secretar excesivas cantidades de estrógenos y una mayor actividad de los granulocitos estromales con un incremento de la secreción de relaxina , agregamos nosotros: por acción de la progesterona o progestágenos que a través de los granulocitos secretarían relaxina, hormona que en último término sería responsable de la RAS, hecho que unificaría los resultados de las dos investigaciones.

En favor de esta afirmación se puede apreciar que la RAS se presentó asociada a endometrio con mayor maduración glandular e índice alto de maduración estromal en donde se presentan granulociTos estromales responsables de la síntesis de relaxina.

Observaciones clínico patológicas en humanos y experimentos controlados en animales inferiores, han conformado 2 grupos de hipótesis sobre la naturaleza de la reacción de Arias-Stella en el endometrio humano.

La primera de ellas sostiene que la RAS es un fenómeno involutivo o regresivo asociado principalmente a aborto espontáneo y a embarazo ectópico, en donde la muerte del embrión o la degeneración del corion se asocia a la consiguiente declinación de las hormonas que soportan el embarazo, a medida que se produce la regresión del cuerpo lúteo.

Previamente, Deelman había sugerido desde antes de la publicación del trabajo clásico de Arias Stella (1954) que el fenómeno era el producto de la combinación de un proceso inflamatorio y degenerativo, afirmado posteriormente por Charles en 1962 (14).

El hallazgo de la RAS durante el embarazo normal objeta seriamente esta hipótesis (5); sin embargo Fienberg y Lloyd explican la aparición del fenómeno como el resultado de los niveles hormonales declinantes a finales del primer trimestre del embarazo, en el momento en que ocurre el relevo entre ovario y placenta (7a-8a semana) (8).

Además el hallazgo de focos de necrosis en la decidua, la picnosis de núcleos, la homogenización de la cromatina y la disminución de los nucleólos en las células de Arias Stella y la descamación de las mismas hacia las luces glandulares constituyeron evidencias histológicas de que se trataba de un fenómeno degenerativo del endometrio.

Sinembargo, otros investigadores apoyan la hipótesis de que la RAS es un fenómeno proliferativo producto de una hiperestimulación hormonal; lo afirman por la evidencia de que la RAS se presenta en embarazos muy tempranos, antes del momento del relevo ovario-placenta. Holmes-Lylle (15) documentaron cambios atípicos compatibles con RAS en un polipo endometrial de una mujer sometida a curetaje y en donde se encontró implantado un embrión de 12 días, siguiendo los criterios de Noyes y Herting, el endometrio fue fechado para el día 23 momento de la máxima actividad del cuerpo lúteo (16).

Oertel (17) revisó la colección de Herting correspondiente a la asociación de embarazos tempranos e histerectomía y encontró RAS en 5 de los 19 especímenes examinados, uno de ellos asociado a un embrión de 17 días.

La mayoría de los cambios histológicos de la RAS, a excepción de los mencionados por Fienberg (8), apuntan a un fenómeno proliferativo secundario a hipersecreción hormonal, específicamente la gran vacuolización del citoplasma y su alto contenido de glicógeno. 
El experimento llevado a cabo por Arias Stella en 1955 mostró que los grupos de ratas normales que recibieron estradiol y HCG y ratas castradas que recibieron estradiol y progesterona en altas dosis, exhibieron cambios atípicos compatibles con la RAS (2).

La ausencia de RAS en el grupo de ratas castradas que recibieron estradiol sólo o en combinación con HCG apoya a la progesterona como mecanismo de hiperestimulación hormonal en la génesis de la RAS.

Por microscopía electrónica se han observado alteraciones citoplasmáticas y nucleares en las células de RAS que indican un estado metabólico activo de las mismas. También se ha observado un pronunciado incremento en el retículo endoplásmico rugoso y en el número de ribosomas que indica una producción activa de proteínas. Las alteraciones nucleares son consistentes con un contenido poliploide del DNA como lo indica el tamaño del núcleo, conservando una relación núcleo-citoplasma normal y la forma de distribución de la cromatina. Estos hallazgos de ultraestructura celular están en contra de un estado involutivo (7-8).

Wagner (19) estudió por microespectrofotometría y cuantificó el DNA en las células de glándulas endometriales con RAS lo que mostró atipismo nuclear como consecuencia de la poliploidia del material cromosómico. Esta duplicación del material genético en las células es causado posiblemente por hiperestimulación hormonal.

En humanos se ha observado la RAS en mujeres sometidas a grandes dosis de estrógenos y progestágenos, es así como Brown en la discusión del trabajo de Roach (6) reporta haber obtenido RAS en mujeres jóvenes oforectomizadas con la administración de una combinación de estrógenos. El mismo Arias Stella (20) menciona experiencias no publicadas, encontrando RAS en el endometrio de mujeres tratadas con valerianato de estradiol y caproato de $17 \mathrm{OH}$ progesterona durante 2 meses.
Otra observación importante hecha en humanos por inmunohistoquímica apunta hacia la HCG como hormona causal directa de la RAS. Vásquez (21) observó RAS en el endometrio en 7 de 11 casos de embarazo ectópico. El tejido fue estudiado aplicando un anticuerpo purificado antiHCG marcado con fluoresceina. Encontró que en las zonas de aparición de la RAS, se hallaba HCG, conforme lo demostraba la fijación selectiva del reactivo inmunofluorescente. Para este autor algunos hallazgos en la histología de la RAS eran semejantes a las reacciones de anafilaxia. Así se plantea la hipótesis de la acción directa y de tipo inflamatorio por parte de la HCG sobre el endometrio en la RAS.

Sin embargo, Ylikorkala (22) no encontró ninguna relación con respecto a los niveles urinarios de HCG entre pacientes que abortaron espontáneamente y la presencia o ausencia de RAS en el endometrio, además en la presente investigación no se encontró mayor frecuencia de RAS en el grupo tratado con HCG parenteral.

La hipótesis de sobreestimulación hormonal observa 3 variantes: a) la hormonodependencia estrogénica y altos niveles de progesterona, b) estrógenos y/o relaxina y c) HCG de forma directa sobre el endometrio.

Con la presente investigación experimental en humanos, se puede concluir que la RAS es un fenómeno proliferativo secundario a estimulación hormonal de una progesterona o un progestágeno, en este caso la $17 \mathrm{OH}$ progesterona, sobre un endometrio proliferativo, dependiente de estrógenos, como lo sugirió Arias Stella en su estudio inicial y posiblemente desencadenando en último término por la relaxina, como se aprecia en los estudios de Dallembach; por lo anterior este fenómeno puede encontrarse en entidades como: persistencia del cuerpo lúteo, folículo luteinizado no roto, ciclos con ovulación múltiple, independientemente de si existe o no un embarazo; además es un fenómeno que se asocia a un estímulo proliferativo y en especial se encuentra en endometrios secretores con mayor índice de maduración, en contra de la opinión opuesta de que es un fenómeno regresivo.

\section{BIBLIOGRAFIA}

1. Arias-Stella J. Atypical endometrial changes associated with the presence of chronic tissue. Arch Pathol. 1954; 58: 112-128.

2. Arias-Stella J. Abnormal endometrial changes induced in the rat. The effects of chronic hormone and estrogen. Arch. Pathol. 1955; 60: 49-58.

3. Dallenbach FD. Experimentelle untersuchungen Zurgenese des AriasStella phanomens. Ver DSTCH Ges Path. 1966; 50: 413.

4. Bernhardt R. Atypical endometrium associated with ectopic pregnancy. Obstet. Gynecol. 1966; 28: 849-853.

5. Silverberg S. Arias Stella phenomenon un spontaneous and therapeutic abortion. Am. J. Obstet. Gynecol. 1969; 103: 828-835.

6. Roach W., Guderian AM., Brewer J. Endometrial gland cell atypism in the presence of trofoblast. Am J Obstet Gynecol. 1960; 79: 680691.

7. De Brux J., Ancla M. Arias Stella endometrial atypias. Cause study with electron microscope. Am. J. Obstet. Gynecol. 1964; 89: 661.
8. Fienberg R., Lloyd HE. Ther Arias-Stella reaction in early normal pregnancy an involutional phenomenon. Human. Pathol. 1974; 5: 183-189.

9. Maroulis G., Abraham G. Ovarian and adrenal contributions to peripheral steroid levels in postmenopausal women. Obstet. Gynecol. 1976; 48: 150-154.

10. Navot D., Lanfer N., Kopolovic J et al. Artificially induced endometrial cycles and establishment of pregnancies in the absence of ovaries. New. Engl. J. Med. 1986; 314: 806-811.

11. Bigazzim, Nardi E., Brani P., Petrucci F. Relaxin in human decidua. J. Clinic Endocrinol Metabol. 1980; 51: 939-947.

12. Irwin Jc., Kirk D., King RJB., Quiglet MM., Gwatkin RB. Hormonal regulation of human endometrial stromal cells in culture: an in vitro model for decidualizacion. Fertil Steril. 1989; 52: 761-768.

13. Rizkallah T. Metabolism of HCG in man. J Clinic Endocrinol. 1969; 5: $92-100$. 
14. Charles MB. The Arias Stella reaction. Obstet. Gynecol. Brit Comm. 1962; 69: 1006-1010.

15. Holmes E., Lylle W. How early in pregnancy does the Arias Stella reaction occur. Arch. Pathol. 1973; 95: 302-303.

16. Noyes RW., Herting AT., Roch J. Dating the endometrial biopsy. Fertil Steril. 1950; 1: 3-25.

17. Oertel Y. THe Arias Stella reaction revisited. Arch. Pathol. 1978; 102: 651-654.

18. Thrasher TV., Richart RM. Ultraestructure of the Arias Stella Reaction. Am. J. Obstet. Gynecol. 1972; 112: 113-120.

19. Wagner D., Richard R. Poliploid in the human endometrium with the Arias Stella reaction. Arch. Pathol. 1968; 85: 475-480.
20. Arias-Stella J. Atypical endometrial changes produced by chronic tissue. Human Pathology 1972; 3: 450-453.

21. Vasquez E. Contribution to the study of the scalled Arias Stella phenomenum by means of inmunohistochemistry. Gynecol. Obstet. Mex. 1972; 32: 1-11.

22. Ylikorkala O., Korhonen M. Relationship between endometrial Arias Stella phenomenon and concentrations of urinary chronic gonadotropic and serum placental lactogen. Act. Obstet. Gynecol. Scand. 1975; 54: 303-305.

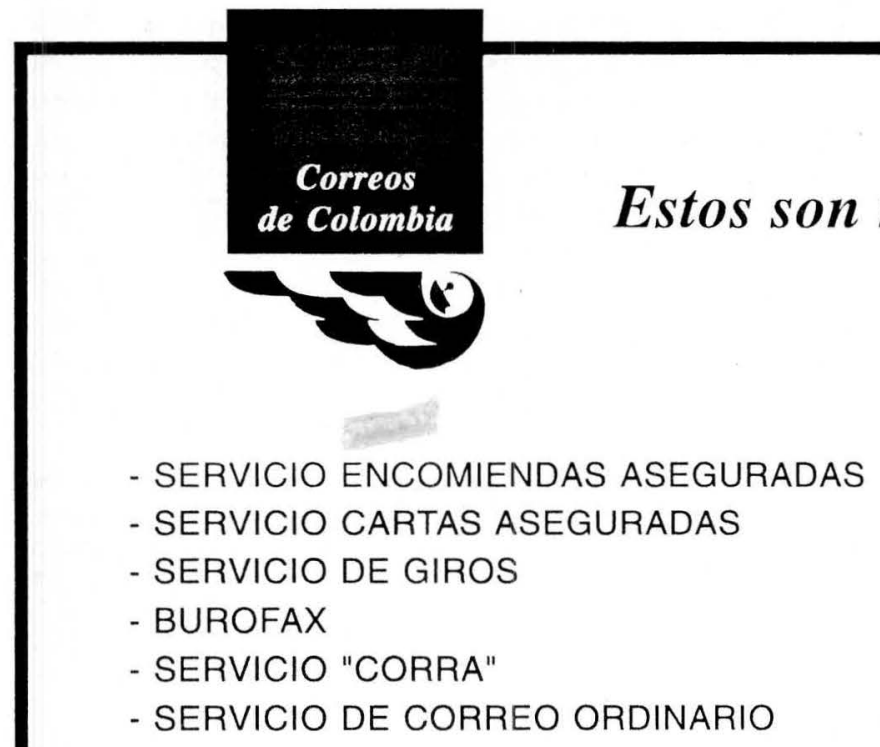

- SERVICIO DE CORREO CERTIFICADO

- SERVICIO DE CERTIFICADO ESPECIAL

- ENCOMIENDAS CONTRA REEMBOLSO

- SERVICIO TARIFA POSTAL REDUCIDA

- SERVICIO DE FILATELIA

- SERVICIO ELECTRONICO

- SERVICIO INTERNACIONAL APR/SAL

- SERVICIO RESPUESTA COMERCIAL

- SERVICIOS ESPECIALES

Teléfonos para quejas y reclamos: 3340304 - 3415536 - Santafé de Bogotá, D.C. - Colombia Cuente con nosotros hay que creer en los Correos de Colombia 